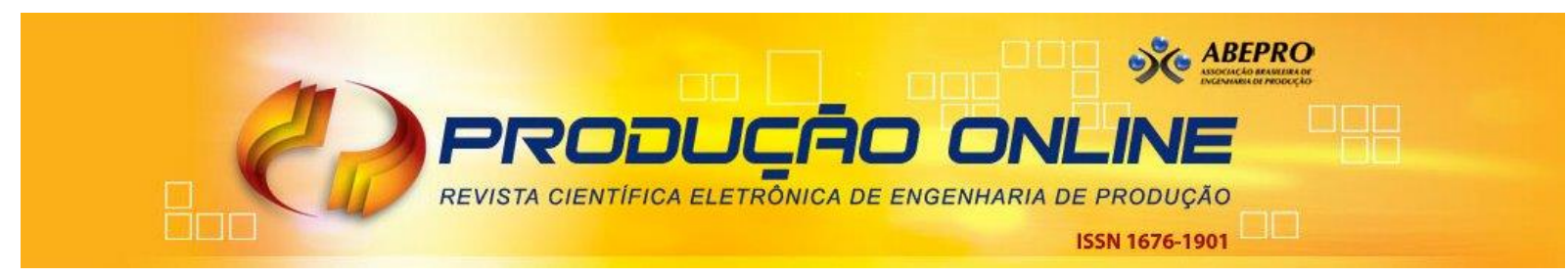

\title{
INTERVENÇÃO ERGONÔMICA E MELHORIA DA PRODUTIVIDADE: É POSSÍVEL?
}

\section{ERGONOMIC INTERVENTION AND PRODUCTIVITY IMPROVEMENT: IS IT POSSIBLE?}

\author{
Gilberto Jesus Avila * E-mail: gilbertojesusa@gmail.com \\ Anderson Braun*E-mail: abraun@feevale.br \\ *Universidade FEEVALE (FEEVALE), Novo Hamburgo, RS
}

\begin{abstract}
Resumo: Este trabalho teve como objetivo aplicar uma Análise Ergonômica do Trabalho (AET) e comparar os resultados de produtividade obtidos antes e depois da aplicação com vistas a demonstrar se após uma implantação de melhorias ergonômicas no ambiente de trabalho ocorre aumento da produtividade. O estudo foi realizado em empresa metalúrgica que produz peças estampadas para a indústria e fabrica produtos de marca própria. Foi explorado o conceito de ergonomia como método para facilitar a interação entre o homem e o seu trabalho dentro de um sistema de produção. Dentre os conceitos de produtividade, foi analisada a Produtividade de Fator Simples (single-factor productivity - SFP). A pesquisa se caracterizou por ser exploratória, teve como base a pesquisa-ação e se orientou pela busca de integração e complementariedade entre a análise qualitativa e a análise quantitativa. Os resultados demonstraram que a produtividade após a implantação da AET aumentou e que os operadores participantes referiram diminuição de dores e desconfortos após as melhorias.
\end{abstract}

Palavras-chave: Ergonomia. Análise Ergonômica do Trabalho - AET. Melhoria. Produtividade. Aumento da produtividade.

Abstract: This study aimed to apply an Ergonomic Work Analysis (EWA) and compare the productivity results obtained before and after the application in order to demonstrate whether after a deployment of ergonomic improvements in the workplace is increased productivity. The study was conducted in a metalworking company that produces stamped parts for the industry and manufactures private label products. It was explored the concept of ergonomics as a method to facilitate interaction between man and his work in a production system. Among the concepts of productivity, it analyzed the Single Factor Productivity - SFP. The research is characterized as exploratory it was based on action research and guided by the search for integration and complementarity between the qualitative analysis and quantitative analysis. The results showed that productivity after implantation EWA increased and operators participating reported decreased pain and discomfort after improvements.

Keywords: Ergonomics. Ergonomic Work Analysis - EWA . Improvement. Productivity. Increased productivity.

\section{INTRODUÇÃO}

Transformar o trabalho é a finalidade central da ergonomia, sendo que essa deve ser aplicada de forma a contribuir para a concepção de situações de trabalho que não alterem a saúde dos trabalhadores e que propiciem o alcance dos objetivos econômicos determinados pela empresa (SEMENSATO, 2013). No entanto, a 
saúde, embora importante, nem sempre é o argumento mais convincente quando comparado à análise de custo/benefício (TOFT; HOWARD; JORGENSEN, 2003). Neste sentido, os empresários almejam uma intervenção ergonômica acompanhada de solução rápida para problemas que causam doenças e afastamentos com, principalmente, muito pouco ou nenhum comprometimento de capital (DEMPSEY; MATHIASSEN, 2006, DEMPSEY, 2007).

Consequentemente, sem a interferência de legislação apropriada, as empresas tendem a serem mais preocupadas em alcançar seus objetivos econômicos do que com a saúde dos trabalhadores (ORMELEZ; ULBRICHT, 2010). No Brasil, por conseguinte, em conjunto com outras, foi criada a Norma Regulamentadora NR17 (BRASIL, 2007) revestida de força de lei, específica para tratar de ergonomia e com o objetivo de estabelecer parâmetros que permitam proporcionar um máximo de conforto, segurança e desempenho eficiente aos trabalhadores.

Para atender tais intenções, métodos conhecidos como Análise Ergonômica do Trabalho (AET) deveriam ser aplicados para adequar ambientes de trabalho já existentes ou a serem construídos (PIZO; MENEGON, 2010, SEMENSATO, 2013). Deste modo, já que a implantação de sistemas de produção ergonomicamente adequados é normalmente vista como despesa (TOFT; HOWARD; JORGENSEN, 2003) ou empecilho ao alcance de objetivos de produção (LIMA, 2000) se estabelece um trade-off a ser equilibrado (PAIVA; CARVALHO Jr; FENSTERSEIFER, 2004).

Neste ponto reside o problema desta pesquisa, o qual é expresso na seguinte pergunta: a produtividade aumenta após a implantação de melhorias no posto de trabalho resultantes da aplicação de uma AET? Espera-se, portanto, que a resposta obtida confirme a hipótese de que após a implantação de melhorias ergonômicas no ambiente de trabalho efetivamente ocorre aumento da produtividade.

Para responder esta questão, o procedimento de pesquisa se orientou pela integração e complementariedade entre a análise quantitativa e a análise qualitativa. A análise quantitativa ocorreu pela aplicação do Método RULA para avaliar a necessidade de melhoria do posto de trabalho e pela análise estatística através do teste $t$ de médias emparelhadas para demonstrar se houve ou não aumento da produtividade. A análise qualitativa, com a finalidade identificar os problemas e buscar as soluções, teve como base a pesquisa-ação.

\section{DESENVOLVIMENTO}

A pesquisa foi organizada obedecendo a seguinte estrutura: inicialmente é feita a análise do referencial bibliográfico abrangendo conceitos sobre ergonomia, sistemas de produção, gestos e posturas críticas, análise ergonômica do trabalho e produtividade. A seção seguinte expõe o método de trabalho. Após se apresenta a coleta e análise de dados. Por fim, são exibidas as considerações finais. 


\subsection{Ergonomia}

A ergonomia é o estudo da adaptação do trabalho ao homem. E examina, portanto, as interações entre pessoas e locais de trabalho para que sejam alcançados os resultados esperados (RABY et al, 2003, IIDA, 2005, PUENTESLAGOS; GARCÍA-ACOSTA, 2011).

A inquietação com as condições da realização do trabalho e suas consequências para os trabalhadores não é recente. No entanto, é a partir de meados do século XX que a atuação de pesquisadores fez com que a ergonomia centrada na análise da atividade fosse destacada, tendo suas bases teóricas aprofundadas e seus métodos enriquecidos com o objetivo de melhorar as condições de trabalho (WILSON, 2000, RABY et al., 2003, PIZO; MENEGON, 2010).

Wilson (2000; 2014), adepto da ergonomia clássica centrada no componente humano dos sistemas homem-máquina, indica que o conceito de ergonomia está alinhado com uma abordagem sistêmica e deve enfatizar a necessidade de um fundamental entendimento da pessoa e suas interações no trabalho e a prática de aprimorar tais interações. Roetting e Luczak (2001), por sua vez, sintonizados com uma abordagem ergonômica focada na atividade humana contextualizada, definem a ergonomia como sendo um conjunto de características técnicas e organizacionais que, de formal saudável, seja capaz de proporcionar produtividade e eficiência.

Para Hendrick (1996), Roetting e Luczak (2001), lida (2005), Dempsey (2007), Semensato (2013) e Sheridan (2014) a ergonomia é classificada como uma ciência associada a áreas do conhecimento tais como tecnologia, saúde e segurança ocupacional e organização social e política do trabalho. É indicado também o caráter interdisciplinar da ergonomia que envolve áreas relativas à medicina, engenharia, psicologia, fisioterapia e administração.

Semensato (2013) sinaliza que estas características são perceptíveis, uma vez que, a abordagem ergonômica em uma organização assume o caráter sistêmico englobando componentes físicos, humanos e tecnológicos. Sagot, Gouin e Gomes (2003) propõem que a ergonomia deve ter foco no estudo da realização das atividades levando em consideração movimentos e gestos e não somente funções operacionais isoladas.

Cabe aqui, então, com o objetivo de vincular sistemas ergonômicos e sistemas de produção, explorar brevemente conceitos sobre estes últimos.

\subsubsection{Ergonomia e Sistemas de Produção}

Bertalanffy (1977) propôs uma teoria geral de sistemas composta por entradas, atividades de processamento, saídas e retroalimentação para monitorar se as saídas atendem os requisitos de entrada. Ohno (1997) conectando esta abordagem à produção definiu sistema de produção como um conjunto de processos e operações utilizados para a obtenção de produtos acabados a partir da 
transformação de matérias primas. Neste sistema, quantidades a serem produzidas e especificações de produto podem ser consideradas como entradas. Informações, aplicação de métodos e recursos podem ser classificadas como atividades de processamento. O produto resultante é a saída do sistema. A retroalimentação ocorre pela verificação de quantidades produzidas e atendimento das especificações de produto.

Associado ao conceito de sistema está o de processo que, por sua vez, inclui o de operação. Assim, um processo pode ser descrito como o fluxo de materiais no tempo e no espaço para a transformação de matéria-prima em componentes semiacabados e após em produto acabado. Operação pode ser definida como o trabalho executado para efetivar a transformação caracterizada pela interação entre equipamentos e operadores (SHINGO, 1996).

De forma análoga, Wilson (2014) propõe que um sistema ergonômico contém na sua essência a interação com pessoas ao invés de se concentrar em uma parte individual, por exemplo, a operação. Sheridan (2014) conceitua sistema ergonômico como sendo a combinação de aspectos comportamentais e ciência biomecânica para análise e desenvolvimento de sistemas técnicos complexos envolvendo pessoas e máquinas e suas interações em um modelo baseado no relacionamento causa-efeito.

lida (2005) enfatiza o caráter interdisciplinar da ergonomia e trata as interações entre o homem e o seu trabalho dentro de um sistema homem-máquinaambiente e associa a abordagem de sistema ergonômico a um modelo de sistema semelhante ao proposto por Bertalanffy (1977). Assim, em tal sistema as entradas são providas por informações oriundas da máquina e do ambiente de trabalho. Estas informações são processadas pelo homem através de seu sistema sensorial e transformadas em ações resultantes de movimentos corporais. As saídas do sistema são os comandos à máquina para realização da operação. A retroalimentação pode ser fornecida por interfaces que orientam as decisões do homem sobre o comportamento do sistema.

Neste contexto, lida (2005) aponta que a análise de sistemas, num nível macro ergonômico, ou seja, aplicado por toda a organização abrange aspectos mais gerais relacionados à realização do trabalho envolvendo a distribuição de tarefas entre homens e máquinas e considerando critérios como custos, confiabilidade e segurança. Robertson (2001) também analisa a harmonia de um sistema macro ergonômico composto por micro sistemas (incluindo interfaces homem-máquina). Assim, aspectos tais como segurança, produtividade e satisfação na realização do trabalho, são positivamente afetados em consequência da sinergia entre os subsistemas.

Esta análise pode, então, ser aprofundada na direção dos postos de trabalho individuais que compõem o sistema, convergindo, assim, para um nível micro ergonômico, uma vez que o posto de trabalho pode ser considerado como um micro sistema contido no sistema total (IIDA, 2005). 
Este trabalho tem a pretensão de propor a convergência entre os aspectos ergonômicos do trabalho e a produtividade por meio do pressuposto de que se há melhoria nas condições ergonômicas para a realização do trabalho, consequentemente ocorre aumento da produtividade. Assim, com o objetivo de buscar esta convergência, a seguir são explorados alguns aspectos da ergonomia relacionados a gestos e posturas críticas.

\subsubsection{Gestos e Posturas Críticas}

Independente do nível de automação que uma empresa apresente, o que faz dela uma fonte de bens e serviços é o trabalhador. Na relação capital-força de trabalho, o trabalhador, para realizar a sua parte, precisa possuir a qualificação e, principalmente, a saúde necessária para execução das tarefas durante o período de trabalho (RIBEIRO, 1999). Portanto, estudar o trabalho só tem sentido quando o trabalhador está inserido no sistema de produção (LIMA, 2000).

Se um trabalhador trabalha é por que precisa trabalhar para prover sua subsistência (RIBEIRO, 1999) e é impossível produzir bem e com qualidade sentindo dor e desconforto (RENNER, 2005). Dor e desconforto que podem ser provocados por doenças ocupacionais conhecidas no Brasil como lesões por esforços repetitivos (LER) e distúrbio osteomusculares relacionados ao trabalho (DORT) (BRASIL, 2000).

Segundo o Ministério da Saúde em protocolo emitido no início dos anos 2000, as LER/DORT são doenças ocupacionais, ou seja, relacionadas ao trabalho (BAGATIN; KITAMURA, 2005) que afetam principalmente os membros superiores. Estas doenças são reconhecidamente polêmicas não só no Brasil (BRASIL, 2000) como também em outros países devido a aspectos variados que podem concorrer para o seu surgimento (SLEATOR; DONNA; GRAHAM, 1998).

Sleator, Donna e Graham (1998), Oliveira (1999), Chiavegato Filho e Pereira Jr. (2004), Renner (2005) e Halim et al. (2012) apontam que fatores relacionados ao trabalho são a causa da existência destas dores referidas por trabalhadores quando executam suas atividades laborais. Portanto, as LER/DORT podem ser atribuídas, por exemplo, a tarefas que se caracterizam por repetição de movimentos, posturas inadequadas, ambiente físico e organização do trabalho.

Além destes fatores, problemas osteomusculares associados à biomecânica, indicam que as LER/DORT também podem se manifestar em outros locais do corpo humano e não somente nos membros superiores. Por outro lado, aspectos antropométricos podem induzir a gestos e posturas inadequados. Assim, o conhecimento destas variáveis pode auxiliar na adaptação do trabalhador ao seu posto de trabalho (RENNER, 2005, BRAUN, 2012).

Consequentemente medidas preventivas deveriam ser implantadas para minimizar ou eliminar gestos e posturas consideradas críticos. Estas medidas deveriam ser voltadas para reeducação postural e gestual não somente para 
melhorias dos postos de trabalho, mas também para adoção de critérios de ergonomia, alinhada com uma visão sistêmica (ROBERTSON, 2001, IIDA, 2005, THEBERGE; NEUMANN, 2010, WILSON, 2014, SHERIDAN, 2014). O capítulo seguinte, que trata da análise ergonômica do trabalho, aprofunda o exame destas medidas.

\subsubsection{Análise Ergonômica do Trabalho - AET}

Semensato (2013) indica que a AET deve necessariamente partir do entendimento do trabalho. De forma semelhante à exposta por Shingo (1996) que apresentou uma definição para operação, Lima (2000), Braun (2012) e Semensato (2013) exploram o conceito de trabalho, a partir dos conceitos de atividade e tarefa.

A atividade pode ser comparada à operação, ou seja, representa a efetivação do trabalho que resulta em um produto ou serviço e caracteriza, por este aspecto, o trabalho real. A tarefa, por sua vez, pode ser explicada como sendo a prescrição do trabalho, isto é, o detalhamento na forma de um plano que engloba o que, quando, como e por quem deve ser executado o trabalho (LIMA, 2000, BRAUN, 2012, SEMENSATO, 2013).

Segundo Lima (2000) somente a análise e o entendimento da atividade é capaz de prover harmonia entre as necessidades de produção e as lógicas conflitantes (por exemplo, necessidades interdepartamentais) para sua realização. A atividade, portanto, pode ser adaptada ou ajustada às condições particulares de cada trabalhador em um mesmo posto de trabalho que possui a determinação da tarefa na forma de procedimentos escritos (SEMENSATO, 2013).

Como resultado, quanto maior for o detalhamento da tarefa, maior será o controle que esta impõe sobre a atividade o que implica em menor variabilidade na sua execução. A ergonomia não visa diminuir a variabilidade, mas sim estuda-la e caracteriza-la, com o objetivo de influenciar nos métodos de execução do trabalho. Assim, a ação ergonômica deve ser focada no trabalho real tendo como base a definição do que deve ser analisado e quão exequível será a intervenção (LIMA, 2000, SEMENSATO, 2013). A variabilidade, por sua vez, está diretamente relacionada à atividade e o seu tratamento deve ocorrer por agentes ligados à organização da produção, porém alinhado com ação ergonômica (LIMA, 2000).

Lima (2000) afirma que a ergonomia e a AET são partes constituintes de uma única metodologia, sendo aquela o conceito fundamental e esta a prática de intervenção. Assim, a análise ergonômica do trabalho tem foco no trabalho efetivamente realizado (LIMA, 2000) e, para tanto, um método apropriado deve ser aplicado (IIDA, 2005).

lida (2005) indica que a AET tem como objetivo desdobrar conhecimentos de ergonomia para analisar, diagnosticar e corrigir uma situação real de trabalho e descreve um método composto por cinco etapas que englobam análise da demanda, análise da tarefa, análise da atividade, diagnóstico e recomendações. Pizo e 
Menegon (2010) propõem um esquema geral da análise ergonômica em sintonia com o método descrito por lida (2005) e enfatizam a necessidade de interação entre o analista e o ambiente de trabalho a ser analisado.

A análise das condições de trabalho referentes às forças, posturas e aos movimentos corporais está vinculada à biomecânica. Assim, a aplicação de métodos voltados para a biomecânica pode se constituir ferramenta com viés quantitativo importante na complementação da análise das condições de trabalho e nas relações entre o homem e suas atividades (IIDA, 2005, BRAUN, 2012).

Os métodos biomecânicos têm como base enfoques fisiológicos (funções orgânicas), psicofísicos (julgamento do esforço percebido) e físicos (descrição de força e movimento) e possuem metodologias específicas para obtenção dos dados e análise dos resultados. Dentre os métodos apresentados por Braun (2012), o método conhecido como Rapid Upper Limb Assesment (RULA) desenvolvido por McAtamney e Corlett (1993) é o procedimento utilizado como complemento da AET foco deste trabalho. Esta técnica foi escolhida por ser de fácil e rápida aplicação no chão de fábrica para uma determinada população de trabalhadores, favorecendo identificar o risco de distúrbios relacionados ao trabalho que afetam os membros superiores.

Até o presente momento foram explorados os principais conceitos inerentes à ergonomia e análise ergonômica do trabalho. Resta examinar a produtividade, o que é apresentado na proporção do interesse desta pesquisa no tópico seguinte.

\subsection{Produtividade}

Nem sempre implantar um sistema para melhorar as condições de trabalho ou a sua organização é a melhor justificativa ou o argumento mais fácil de ser aceito por aqueles que têm a responsabilidade pela tomada de decisão. Assim como Juran e Gryna (1991) sinalizam que a linguagem do dinheiro é essencial para o gerenciamento da qualidade, na abordagem ergonômica, Hendrick (1996) lembra que é fundamental demonstrar resultados que indiquem melhorias de competitividade e manutenção da sobrevivência da empresa.

Portanto, se torna importante quantificar as melhorias nas condições de operação resultantes da aplicação de análises ergonômicas do trabalho. Consequentemente, um indicador que aponte que a produtividade é positivamente influenciada pela intervenção ergonômica poderia ser mais confiável e, principalmente, melhor entendido pelos tomadores de decisão quando se aborda a relação custo-benefício (HENDRICK, 1996, VINK; KONINGSVELD; MOLENBROEK, 2006, DEMPSEY, 2007).

Tangen (2002), Poloni e Silva (2010) e Syverson (2011) propõem que a produtividade representa a eficiência com que ocorre a transformação de entradas (recursos empregados) em saídas (produtos produzidos). Campos (1992), em sintonia com esta definição, apresenta esta eficiência na sua forma matemática mais 
simples e explica que a produtividade, então, é o quociente entre o que uma empresa produz e o que ela consome para produzir.

A produtividade, dependendo do ponto de vista, pode ser conceituada de formas diferentes para alcançar objetivos específicos. Com este enfoque, por exemplo, pode-se afirmar que quanto maior a produtividade, maior valor agregado terá o produto resultante da transformação das entradas em saídas. Por outro lado, a produtividade, quando vinculada estritamente à atividade de produção no chão de fábrica, pode ser classificada em alguns tipos de acordo com a finalidade da medição (GUNASEKARAN; CECILLE, 1998, TANGEN, 2002, SYVERSON, 2011).

Dentre as formas mais comuns de medição da produtividade na indústria, Gunasekaran e Cecille (1998) e Syverson (2011) destacam a produtividade de fator simples (single-factor productivity - SFP), que é a de interesse deste trabalho. Esta produtividade relaciona a quantidade produzida de um produto a um único recurso utilizado na sua produção e tem como base o quociente entre grandezas físicas. Assim, é possível medir a quantidade de peças produzidas em um determinado período de tempo definido em horas ou minutos. O resultado, por exemplo, poderia ser expresso em peças por hora.

Aqui vale enfatizar o viés de subjetividade relacionado a uma medida de produtividade. Homans (1941) sinalizou que em uma pesquisa com vistas a mensurar se a produtividade é afetada pelo ambiente de trabalho, o desempenho do operador pode ser influenciado pelo sentimento de controle. Em sintonia com esta abordagem, Theberge e Neumann (2010), indicam que o alcance de melhorias ergonômicas no local de trabalho e o aumento da credibilidade nas intervenções podem ser influenciados pela presença do ergonomista.

Neste ponto, mesmo com a ressalva do parágrafo anterior, pode ser relembrado o trade-off anteriormente apresentado. Espera-se, portanto, que a produtividade seja a chave para se alcançar a harmonia entre a ação ergonômica e a organização da produção. Isto poderá ser verificado mais tarde no tópico que trata da análise de dados e resultados deste trabalho. Porém, antes, no próximo tópico, é feita abordagem do método de pesquisa.

\subsection{Método de pesquisa}

Quanto aos fins/objetivos este trabalho se caracteriza por ser exploratório (MALHOTRA, 2001, PRODANOV; FREITAS, 2013) e com relação aos meios/procedimentos este estudo teve como base a pesquisa-ação (THIOLLENT, 1997). Na pesquisa-ação, com a finalidade identificar os problemas e buscar as soluções, o pesquisador se insere, de forma planejada, no universo pesquisado não somente como observador, mas também como sujeito ativo. Neste procedimento é detectado o problema e seus agentes, se identifica, discute e se aplica os instrumentos de coleta de dados. É feito monitoramento e se pode atuar no sentido de corrigir as ações na direção dos objetivos. 
Relativamente à abordagem, a pesquisa se orientou pela busca de integração entre a análise qualitativa e a análise quantitativa (BRYMAN, 1988). A pesquisa quantitativa tem como característica principal analisar e traduzir os resultados sob a forma numérica por meio de técnica estatística apropriada. A análise quantitativa ocorreu por meio da aplicação do Método RULA (MCATAMNEY; CORLETT, 1993) para avaliar a necessidade de melhoria do posto de trabalho e através do teste $t$ de médias emparelhadas (com o uso do software estatístico SPSS) para demonstrar se houve ou não aumento da produtividade (MALHOTRA, 2001, ANDERSON; SWEENEY; WILLIAMS, 2003).

A pesquisa qualitativa, por sua vez, se distingue por ser uma metodologia na qual o ambiente em que se desenvolve o estudo é a fonte direta para coleta de dados e a amostra extraída para análise é pequena (MALHOTRA, 2001, PRODANOV; FREITAS, 2013). O instrumento utilizado para a pesquisa qualitativa foi um roteiro de entrevista. As entrevistas foram gravadas e as respostas foram descritas e foi aplicada a técnica de análise explicativa do conteúdo para deixar mais claro o teor do material coletado. Para validar a descrição, o texto resultante foi submetido à aprovação dos entrevistados (FLICK, 2004).

\subsubsection{Unidade de Análise}

A empresa objeto deste estudo é uma metalúrgica que produz peças estampadas sediada em Eldorado do Sul, RS e que atua nos segmentos de fornecimento de peças para a indústria e na fabricação de produto de marca própria. A etapa de produção estudada na empresa foi a estampagem de uma peça que compõe o produto de marca própria. Esta peça é produzida em uma prensa excêntrica dedicada e adaptada para esta operação e operada por um único operador.

\subsubsection{Técnicas de Coleta de Dados}

Conforme proposto por lida (2005) e Pizo e Menegon (2010), para aplicação da AET foram seguidos os seguintes passos: análise da demanda, análise da tarefa e atividades, diagnóstico e recomendações. A aplicação destas etapas teve como base a sequência empregada por Hembecker e Rebeschini (2006).

\subsection{Coleta e Análise dos Dados}

A coleta e análise dos dados foram desenvolvidas em três momentos distintos: antes da aplicação da AET, durante a aplicação da AET e após a aplicação da AET. Estas três etapas são detalhadas a seguir. 


\subsubsection{Antes da Aplicação da AET}

Esta etapa da pesquisa foi composta pela definição e descrição da operação. Em seguida é apresentada a análise da produtividade antes da AET.

A escolha sobre qual seria a operação a ser analisada resultou da observação das operações realizadas na área de produção da empresa. Dentre as operações, foi definida a operação da peça denominada de tirante médio. Esta peça é produzida em uma prensa excêntrica dedicada e adaptada para esta operação na qual o operador se mantém sentado.

A matéria prima é constituída de tira de aço proveniente de uma bobina que é montada em um dispositivo giratório que propicia ao operador puxar a tira sobre uma calha, desenrolando a bobina e introduzindo a tira no dispositivo da prensa. A tira de aço atravessa o dispositivo passando por dentro da ferramenta sendo posicionada em batente fixado na lateral oposta da prensa. A Figura 1 apresenta a prensa e a indicação dos locais onde são feitas a introdução da tira e a retirada da peça.

\section{Figura 1 - prensa excêntrica utilizada para a peça tirante médio}

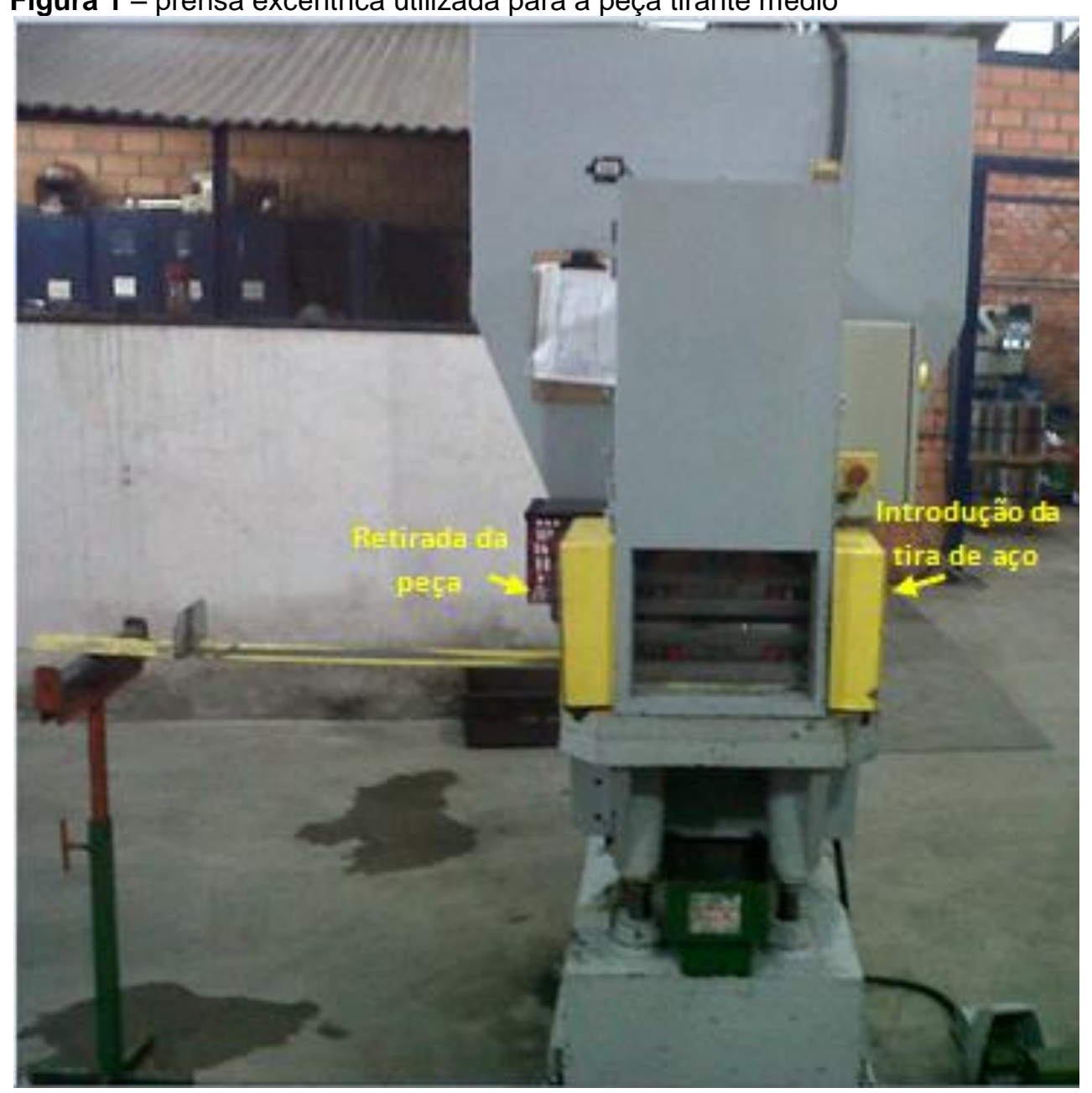

Fonte: Elaborado pelo autor 
A peça é produzida em duas operações em duas prensas distintas. A operação estudada foi a primeira que faz simultaneamente o corte da tira de aço e uma parte da conformação. A segunda operação realiza a finalização da conformação.

A primeira operação foi escolhida levando em consideração o critério de maior exigência física por parte do operador. Nesta operação o operador primeiro manuseia a tira de aço e depois manuseia a peça cortada resultante do acionamento da prensa. Para introduzir a matéria prima na ferramenta da prensa o operador puxa a tira de aço com a mão direita. Para posicionar a matéria prima na posição de corte e conformação é necessário utilizar ambas as mãos. O acionamento do martelo é feito por pedal. Após o corte e a conformação, a peça é colocada na caixa de peças prontas.

\subsubsection{Análise da Produtividade}

Para análise da produtividade antes da AET foram obtidas as folhas de operação compreendidas entre o período de fevereiro até setembro de 2014. Nesta análise, o interesse esteve voltado para a determinação da produtividade de fator simples definida em peças por hora. Para tanto, foi realizada uma análise descritiva com o objetivo de quantificar o numero total de peças produzidas e o tempo total utilizado para produção destas peças por operador e a estatística resultante foi a produtividade por operador.

Durante o período de produção analisado, um total de oito operadores executou a operação da peça totalizando vinte e três ciclos de produção e foram escolhidos os operadores com a maior e com a menor produtividade para a aplicação da $A E T$. Os operadores, portanto, que se enquadraram neste critério foram o OPERADOR 3 e o OPERADOR 8.

O OPERADOR 3 tem altura de $1 \mathrm{~m} 70 \mathrm{~cm}$ e o OPERADOR 8 mede $1 \mathrm{~m} 86 \mathrm{~cm}$. O Quadro 1 abaixo, sumariza os dados encontrados para estes operadores.

Quadro 1 - produtividade do OPERADOR 3 e do OPERADOR 8
\begin{tabular}{|l|l|l|l|}
\hline OPERADOR & $\begin{array}{l}\text { Quantidade } \\
\text { produzida }\end{array}$ & $\begin{array}{l}\text { Tempo efetivo de } \\
\text { produção (horas) }\end{array}$ & $\begin{array}{l}\text { Produtividade } \\
\text { (peças/hora) }\end{array}$ \\
\hline 3 & 6640 & 26,17 & 253,8 \\
\hline 8 & 4012 & 26,50 & 151,4 \\
\hline
\end{tabular}

Fonte: elaborado pelo autor

Analisando-se o Quadro 1 é possível comparar as produções de ambos os operadores. O OPERADOR 3 produziu uma quantidade maior de peças. Os tempos efetivos de produção são semelhantes e a produtividade do OPERADOR 3 é maior que a do OPERADOR 8. 
Estas análises até aqui apresentadas objetivaram contextualizar a operação antes da aplicação da AET. A seção a seguir trata da aplicação da AET.

\subsubsection{Aplicação da AET}

Nesta etapa foram realizadas as entrevistas aos operadores escolhidos, foi observada a execução da operação com registros fotográficos e foi aplicado o método RULA.

Para realização das entrevistas foi utilizado um roteiro de entrevista. Este roteiro possibilita registrar a altura do entrevistado e possui as perguntas relativas à operação. Existem perguntas sobre se são sentidas dores nas mãos e punhos, braços e ombros, pescoço e nas costas e pernas e pés. Também há questões sobre desconfortos abordando esforços de flexão do tronco, levantamento de pesos, movimentos bruscos, formigamentos nas pernas e sentimento de cansaço. $O$ roteiro ainda contém perguntas sobre se ocorrem revezamentos entre operadores durante a jornada de trabalho e se ocorrem intervalos para descanso.

Com relação ao sentimento de dores, todas as respostas dos operadores foram positivas. Para as questões sobre desconfortos, todas as respostas foram negativas, com exceção da questão relativa ao sentimento de formigamentos nas pernas. Para esta, o OPERADOR 3, respondeu que sentia formigamentos devido à pressão sobre o músculo posterior da coxa causada pela madeira do acento da cadeira exposto na borda externa. Por fim, para as questões sobre revezamentos e intervalos para descanso, as respostas foram negativas.

A etapa de observação da execução da operação e os registros fotográficos teve como base a aplicação do método RULA. Foi fotografada a realização da operação por parte dos dois operadores, um de cada vez. Foram produzidas fotografias mostrando a execução da operação pelos lados direito e esquerdo e também pelas costas do operador. Depois desta fase, foi feita análise dos ângulos sobre as fotografias para braços e punhos, pescoço e tronco. De posse dos ângulos obtidos, foi feita a análise do nível de ação através da aplicação da planilha de cálculo do método RULA.

Para o OPERADOR 3 a pontuação resultante foi igual a cinco que está dentro da faixa do nível de ação três, indicando que mudanças devem ser implantadas o mais rápido possível. Para o OPERADOR 8 a pontuação foi igual a sete. Este resultado ficou dentro da faixa do nível de ação quatro e aponta que mudanças devem ser implantadas imediatamente.

$\mathrm{Na}$ seção a seguir, que aborda as análises após a aplicação da $A E T$, são apresentados o diagnóstico e as recomendações de melhorias. 


\subsubsection{Análises Após Aplicação da AET}

Depois da aplicação do método RULA, ocorreram as etapas de diagnóstico e recomendações de melhorias. Assim, durante o diagnóstico e discussão envolvendo os participantes da pesquisa-ação conforme proposto por Thiollent (1997), foram avaliadas as possibilidades de melhorias a serem implantadas.

As análises tiverem como foco duas linhas de melhorias. A primeira voltada para alteração do método de produção e configuração da máquina e dispositivos auxiliares. A segunda foi direcionada para interferência na postura na execução da tarefa por parte do operador. A primeira linha de melhoria, por questões de custos, foi rejeitada.

Assim, foi proposto, e escolhido, um método voltado à correção da postura do operador. Este método, mais simples e praticamente sem investimento de capital, consistiu da fixação de um espelho estreito na parte frontal da máquina possibilitando ao operador enxergar somente os próprios olhos. O pressuposto para este critério reside no fato de que para enxergar os olhos no espelho estreito seria necessário um melhor alinhamento do pescoço, ombros e tronco impedindo inclinações prejudiciais durante a operação. Além disto, para realizar o ajuste da altura para avistar os olhos no espelho, foi proposto o uso de uma cadeira com altura apropriada.

Depois de fixado o espelho na prensa, conforme apresentado na figura 2, foram escolhidas as cadeiras mais apropriadas para o OPERADOR 3 e para 0 OPERADOR 8 , devido às diferenças de altura de ambos. Foi feita orientação sobre a melhor postura durante a operação e, após testar as posturas, as cadeiras foram identificadas por operador. Em seguida, foi determinado a cada operador que somente utilizasse a sua cadeira para operar a máquina. 
Figura 2 - espelho fixado na prensa

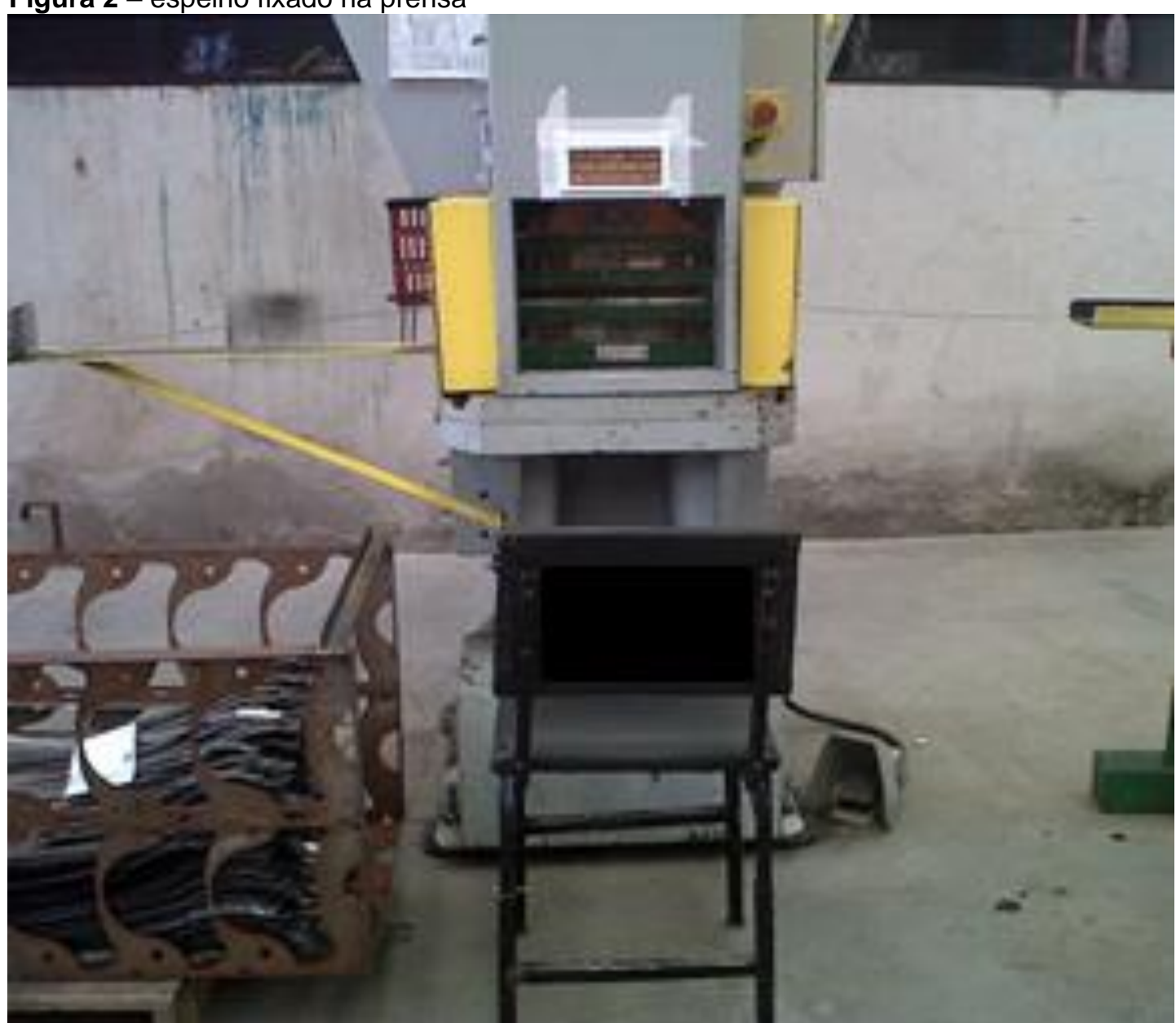

Fonte: Elaborado pelo autor

Para a melhoria relativa à interferência na execução da tarefa por parte do operador, foi proposta uma alteração na execução da operação com o objetivo de minimizar os efeitos do tempo prolongado a que o operador estava submetido a uma mesma posição durante a produção. Foi definido, então, um método de pausas e também de revezamentos com registro na folha de operação.

A próxima seção aborda a repetição do método RULA após a implantação destas melhorias propostas.

\subsubsection{Aplicação do Método RULA Após as Melhorias}

Depois de implantadas as melhorias foram novamente observadas $e$ fotografadas as operações da peça e foi aplicado o método RULA. Novamente foi fotografada a operação pelos lados direito e esquerdo e também pelas costas do operador.

Como resultado das análises, para o OPERADOR 3 a pontuação foi igual a quatro, melhor que a anterior cujo resultado fora cinco. Para o OPERADOR 8, por sua vez, a pontuação foi igual a quatro, igualmente melhor que a anterior que havia 
sido igual a sete. Estes resultados estão dentro da faixa do nível de ação dois e indica que as investigações sobre as posturas devem prosseguir e que melhorias são necessárias, porém não imediatamente.

\subsubsection{Análises Após as Melhorias}

Nesta etapa foi realizada a comparação entre a produtividade antes e depois da AET, foi aplicada a análise estatística através do teste $t$ de médias emparelhadas e os operadores foram novamente entrevistados.

Para análise da produtividade depois da aplicação da AET, com o objetivo de se atender o mesmo critério aplicado antes da AET, as folhas de operação que contemplaram o total de peças produzidas por operador no período de outubro a dezembro de 2014 foram avaliadas. Nestes documentos foi possível verificar registros de pausas durante a produção, porém não se verificaram registros de revezamentos conforme havia sido proposto. O Quadro 2 apresenta os resultados para estas medições.

Quadro 2 - produtividade dos operadores depois das melhorias

\begin{tabular}{|l|l|l|l|}
\hline OPERADOR & $\begin{array}{l}\text { Quantidade } \\
\text { produzida }\end{array}$ & $\begin{array}{l}\text { Tempo efetivo de } \\
\text { produção (horas) }\end{array}$ & $\begin{array}{l}\text { Produtividade } \\
\text { (peças/hora) }\end{array}$ \\
\hline 3 & 13448 & 49,25 & 273,1 \\
\hline 8 & 6207 & 30,83 & 201,3 \\
\hline
\end{tabular}

Fonte: elaborado pelo autor

O Quadro 2 possibilita comparar os dados referentes aos operadores. Novamente o OPERADOR 3 realizou a produção da peça por um período maior de tempo e a quantidade produzida também é maior que a do OPERADOR 8. A produtividade do OPERADOR 8 ainda é menor, porém numa proporção menor.

No entanto, a comparação mais importante neste momento, deve ser feita entre a produtividade antes da AET e depois da AET. O Quadro 3, a seguir, apresenta esta comparação.

Quadro 3 - produtividade antes e depois da AET

\begin{tabular}{|c|c|c|c|}
\hline \multirow{2}{*}{ OPERADOR } & \multicolumn{3}{|c|}{ PRODUTIVIDADE (peças/hora) } \\
\cline { 2 - 4 } & Antes da AET & Depois da AET & Diferença para mais \\
\hline 3 & 253,8 & 273,1 & 19,3 \\
\hline 8 & 151,4 & 201,3 & 49,9 \\
\hline
\end{tabular}

Fonte: elaborado pelo autor

O Quadro 3, portanto, demonstra que ocorreu aumento da produtividade por operador depois de implantadas as melhorias propostas. No entanto, com a finalidade de prover confiança estatística para esta conclusão, foi empregado o teste 
$t$ de médias emparelhadas. Esta estatística, que é aplicável para pequenas amostras, compara as médias das amostras aos pares (MALHOTRA, 2001, ANDERSON; SWEENEY; WILLIAMS, 2003). Assim, devido ao pequeno tamanho da amostra e para garantir a força do teste estatístico, foi escolhido o nível de significância de 0,05 para demonstrar que existem diferenças entre as médias (HAIR et al., 1998, MALHOTRA, 2001). Para o tratamento estatístico destes dados foi utilizado o software SPSS.

Durante a realização da análise estatística, foi verificado que a amostra inicial para os operadores treinados antes da $\mathrm{AET}$ era muito pequena (somente onze casos) e, portanto, a significância estatística após a aplicação do teste $t$ de médias emparelhadas poderia ser prejudicada. Por este motivo, decidiu-se por comparar a produtividade de todos os operadores antes da AET à produtividade dos operadores que participaram das melhorias depois da AET. Desta forma, a amostra dos operadores após a aplicação da AET foi aumentada até um tamanho igual à soma de todos os operadores antes da AET, ou seja, vinte e três casos. O Quadro 4 apresenta estas produtividades.

Quadro 4 - comparação das produtividades antes e depois da AET

\begin{tabular}{|c|c|c|c|c|c|c|c|c|}
\hline \multicolumn{7}{|c|}{ PRODUTIVIDADE (peças/hora) } \\
\hline $\mathbf{n}$ & Antes & Depois & $\mathbf{n}$ & Antes & Depois & $\mathbf{n}$ & Antes & Depois \\
\hline 1 & 187,7 & 260,0 & 9 & 188,2 & 265,7 & 17 & 300,2 & 152,7 \\
\hline 2 & 169,2 & 171,4 & 10 & 204,0 & 227,8 & 18 & 397,6 & 171,4 \\
\hline 3 & 139,5 & 375,0 & 11 & 200,2 & 287,5 & 19 & 63,6 & 200,0 \\
\hline 4 & 221,3 & 312,0 & 12 & 173,33 & 273,2 & 20 & 200,0 & 193,8 \\
\hline 5 & 252,0 & 285,7 & 13 & 232,0 & 285,2 & 21 & 99,3 & 248,8 \\
\hline 6 & 223,0 & 162,2 & 14 & 300,0 & 300,0 & 22 & 135,3 & 186,0 \\
\hline 7 & 160,50 & 357,1 & 15 & 224,6 & 203,4 & 23 & 90,3 & 287,7 \\
\hline 8 & 191,84 & 318,3 & 16 & 257,14 & 192,8 & -- & -- & --- \\
\hline
\end{tabular}

Fonte: elaborado pelo autor

Para aplicação do teste $t$ foi testada e confirmada a normalidade da distribuição das amostras através do teste de Shapiro-Wilk (HAIR et al., 1998, ANDERSON; SWEENEY; WILLIAMS, 2003, TORMAN; COSTER; RIBOLDI, 2012). Os dados antes da AET apresentaram significância igual a 0,563 e a amostra após a $A E T$ apresentou significância de 0,286 . Desta forma, ambos os resultados ficaram acima do nível de 0,05 que é a condição de normalidade. Em seguida foi aplicado o teste $t$ de médias emparelhadas, apresentado no Quadro 5, que foi compilado do software SPSS. 
Quadro 5 - teste t de médias emparelhadas

\begin{tabular}{|c|c|c|c|c|c|c|c|c|c|}
\hline \multicolumn{10}{|c|}{ Teste $t$ de médias emparelhadas } \\
\hline & & \multirow[t]{2}{*}{$\begin{array}{l}\frac{\pi}{0} \\
\stackrel{\mathbb{d}}{\Sigma}\end{array}$} & \multirow[t]{2}{*}{ 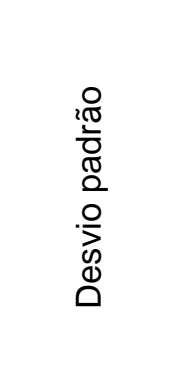 } & \multirow[t]{2}{*}{$\begin{array}{l}\frac{20}{2 \pi} \\
\frac{10}{0} \\
\frac{\pi}{0} \\
0 \\
\stackrel{0}{\frac{2}{4}}\end{array}$} & \multicolumn{2}{|c|}{ 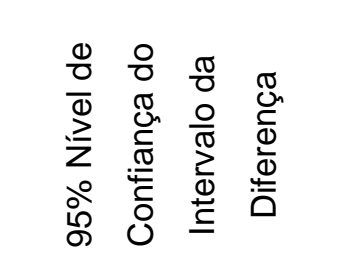 } & \multirow[t]{2}{*}{$\begin{array}{l}\frac{1}{0} \\
\frac{0}{0} \\
\frac{0}{0} \\
>\end{array}$} & \multirow{2}{*}{ 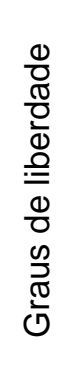 } & \multirow{2}{*}{ 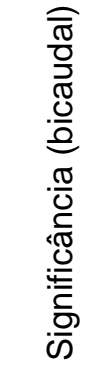 } \\
\hline & & & & & Inferior & Superior & & & \\
\hline $\begin{array}{c}\text { Par } \\
1\end{array}$ & $\begin{array}{c}\text { DEPOIS } \\
- \\
\text { ANTES }\end{array}$ & 48,1313 & 109,16506 & 22,76249 & 0,92479 & 95,33782 & 2,115 & 22 & 0,046 \\
\hline
\end{tabular}

Fonte: elaborado pelo autor

No Quadro 5 é possível verificar que existem diferenças estatisticamente significativas entre as médias. A significância do teste apresentou resultado igual a 0,046, próxima do limite, mas abaixo do nível de significância de 0,05. É possível afirmar, então, com um nível de confiança maior que $95 \%$, que a produtividade após a AET aumentou, confirmando a hipótese anteriormente formulada.

Encerrada esta análise, o próximo passo para atender a complementariedade entre a abordagem quantitativa e qualitativa é a apresentação dos resultados das entrevistas com os operadores após a AET.

Assim, após as melhorias, foram feitas entrevistas com os operadores através da aplicação do roteiro e método anteriormente descritos. As entrevistas foram gravadas e o texto resultante foi submetido à aprovação dos entrevistados.

Com relação às dores anteriormente sentidas, os dois operadores relataram que ocorreu diminuição. Para as questões relacionadas ao desconforto as respostas foram negativas, inclusive para o sentimento de formigamentos, uma vez que as cadeiras que passaram a ser utilizadas possuíam assentos melhores.

\section{CONSIDERAÇÕES}

Este estudo teve como objetivo verificar se após uma implantação de melhorias ergonômicas, resultantes de uma AET, efetivamente ocorre aumento da produtividade. Para tanto, para o posto de trabalho pesquisado, foram analisadas ergonomia e produtividade antes da aplicação da AET. Em seguida foi aplicada a AET e foram implantadas as melhorias resultantes da análise. Por fim, foi verificado se ocorreu ou não aumento da produtividade após as melhorias.

Os conceitos e técnicas associados à ergonomia e à análise ergonômica do trabalho foram explorados na revisão bibliográfica e isto proveu o embasamento necessário para aplicação dos passos da AET e do método RULA. Para o estudo foi definido o posto de trabalho composto por prensa excêntrica e os operadores para 
aplicação da $A E T$ e foi medida a produtividade deste posto por um período de tempo antes da AET.

Como resultado, os operadores escolhidos foram entrevistados. As entrevistas revelaram o sentimento de dores e desconfortos, o que foi confirmado pela aplicação do método RULA que indicou a necessidade de melhorias das posturas. Depois desta etapa, foi definida a melhoria a ser implantada. Dentre as possibilidades estudadas, foi implantada uma melhoria voltada para correção da postura de operação por meio da instalação de um espelho na prensa como referência de altura e escolha da cadeira a ser utilizada na operação.

Novamente foi aplicado o método RULA e os resultados indicaram melhorias nas posturas para execução da operação. Após novo ciclo de produção nesta condição melhorada foi feita nova medição da produtividade. Para comparação da produtividade antes e depois da AET foi empregado $o$ teste $t$ de médias emparelhadas. Os resultados demonstraram com significância estatística que a produtividade após a implantação da AET aumentou. Por fim, foram entrevistados os operadores e foram referidas diminuição de dores e desconfortos após as melhorias.

No entanto, durante a realização desta pesquisa foram encontradas algumas dificuldades. A aplicação do método RULA foi satisfatória, porém, não foi possível fazer um acompanhamento constante das operações na condição melhorada, sendo este acompanhamento delegado à coordenação da produção e isto pode ter contribuído para uma carência de disciplina no cumprimento dos procedimentos. Isto foi observado nos registros nas folhas de operação que apresentaram períodos de produção sem revezamentos, conforme havia sido planejado. Por outro lado, é importante salientar que a produção sob observação também é um fator que pode ter influência nos resultados.

Por fim, o tamanho da amostra dos ciclos de produção dos operadores pesquisados antes da AET se apresentou pequena. Foi necessária uma compensação através do aumento da amostra dos ciclos de produção depois da AET destes operadores e comparação com o conjunto dos ciclos de produção de todos os operadores antes da AET.

Apesar destes contratempos, conclui-se que os objetivos foram alcançados. Como recomendações para futuras pesquisas se sugere, conforme indicado pelo método RULA após a melhoria implantada, prosseguir na adequação de melhorias nos postos de trabalho. Isto pode ser obtido através da implantação de algumas das alternativas que foram descartadas como, por exemplo, a alteração do método de produção e configuração da máquina e dispositivos auxiliares por meio de aplicação de alimentador automático para a matéria prima. 


\section{REFERÊNCIAS}

ANDERSON, David R.; SWEENEY, Thomas A.; WILLIAMS, David R. Estatística aplicada à administração e economia. São Paulo: Pioneira Thomson Learning, 2003. 642 p.

BAGATIN, ERICSON; KITAMURA, SATOSHI. História ocupacional. Jornal Brasileiro de Pneumologia, São Paulo, maio 2006, v. 32 (Supl1), S12-S16. Disponível em: < http://www.scielo.br/scielo.php?pid=1806-371320060008\&script=sci_issuetoc> Acesso em: 18 maio 2014.

BERTALANFFY, Ludwig Von. Teoria geral dos sistemas. 3. ed. Petrópolis: Vozes, 1977. $351 \mathrm{p}$.

BRASIL. Ministério do Trabalho e Emprego. NR17 - Ergonomia. Brasília: Ministério do Trabalho e Emprego, 2007. Disponível em:

<http://portal.mte.gov.br/data/files/FF8080812BE914E6012BEFBAD7064803/nr_17.pdf> Acesso em: 29 março 2014.

BRASIL. Ministério da Saúde. Protocolo de investigação, diagnóstico, tratamento e prevenção de lesão por esforços repetitivos/distúrbios osteomoleculares relacionados ao trabalho/secretaria de políticas de saúde. Brasília: Ministério da Saúde, 2000. Disponível em: <http://www.ergonomianotrabalho.com.br/ler_dort_protocolo.pdf> Acesso em: 12 abril 2014.

BRAUN, Anderson. Ergonomia aplicada ao projeto mecânico assistido por computador. 2012. 99 f. Monografia (Trabalho de conclusão do Curso de Pós Graduação em Engenharia de Segurança do Trabalho) - Universidade FEEVALE, Novo Hamburgo 2012.

BRYMAN, Alan. Quantity and quality in social research. Londres: Unwin Hyman, 1988. $198 \mathrm{p}$.

CAMPOS, Vicente Falconi, TQC: controle da qualidade total (no estilo japonês). 6. ed. Belo Horizonte: Fundação Cristiano Ottoni, 1992. 229 p.

CHIAVEGATO Filho, Luiz Gonzaga; PEREIRA Jr, Alfredo. LER/DORT: multifatorialidade etiológica e modelos explicativos. Interface - Comunic., Saúde, Educ., Botucatu, v.8, n.14, p.149-62, set.2003-fev.2004, Disponível em:

<http://www.scielo.br/pdf/icse/v8n14/v8n14a08.pdf> Acesso em: 1 maio 2014.

DEMPSEY, Patrick G. Effectiveness of ergonomics interventions to prevent musculoskeletal disorders: beware of what you ask. International Journal of Industrial Ergonomics, Amsterdam, v. 37, issue 2, p. 169-173, february 2007.

DEMPSEY, Patrick G.; MATHIASSEN, Svend E. On the evolution of task-based analysis of manual materials handling, and its applicability in contemporary ergonomics. Applied Ergonomics, Hopkinton, v. 37, p. 33-43, 2006. DOI: http://doi:10.1016/j.apergo.2004.11.004

FLICK, Uwe. Uma introdução à pesquisa qualitativa. Porto Alegre: Bookman, 2004. 408 p.

GUNASEKARAN, A.; CECILLE, P. Implementation of productivity improvement strategies in a small company. Technovation, Uxbridge, v. 18, n. 5, p. 311-320, 1998. Disponível em: 
$<$ https://www.umassd.edu/media/umassdartmouth/businessinnovationresearchcenter/publica tions/productivity_smes_casestudy.pdf> Acesso em: 28 março 2014.

HAIR, Joseph F.; ANDERSON, Ronald L.; TATHAM, Rolph E.; BLACK, William C. Multivariate data analysis. 5. ed. New York: Prentice-Hall, 1998. 768 p.

HALIM, Isa; OMAR, Abdul Rahman; SAMAN, Alias Mohd; OTHMAN, Ibrahim. Assessment of muscle fatigue associated with prolonged standing in the workplace. Saf Health Work, Bethesda, V. 3, n. 1, p. 31-42, mar. 2012. Disponível em: <http://www.ncbi.nlm.nih.gov/pmc/articles/PMC3430927/> Acesso: 31 mar. 2014.

HEMBECKER, Paula K.; REBESCHINI, Shirley Vargas P. Análise do risco de lesão musculo-esquelética pelo metodo RULA - Rapid Upper Limb Assessement - em trabalhadores de faturamento hospitalar. In: $14^{0}$ Congresso Brasileiro de Ergonomia $/ 4^{0}$ Forum Brasileiro de Ergonomia/2 ${ }^{0}$ ABERGO Jovem/II Congresso Brasileiro de Iniciação em Ergonomia, 2006. Anais... Curitiba, 2006.

HENDRICK, Hal W. Good Ergonomics Is Good Economics. In: 40th Annual Meeting of the Human Factors and Ergonomics Society, 1996, Santa Monica. Proceedings of the Human Factors and Ergonomics Society, Santa Monica, 1996. p. 1-15.

HOMANS, George C. Fatigue of workers, its relation to industrial production. New York: Reinhold, 1941. 165 p.

IIDA, Itiro. Ergonomia: projeto e produção. 2. ed. São Paulo: Blucher 2005. 614 p.

JURAN, Joseph M.; GRYNA, Frank M. Controle da qualidade. São Paulo: Makron Books, 1991. 377 p.

LIMA, Francisco de Paula A. Ergonomia e projeto organizacional: a perspectiva do trabalho. PRODUÇÃO, Rio de Janeiro, n. especial, p. 71-98, 2000.

MALHOTRA, Naresh K.., Pesquisa de marketing: uma orientação aplicada. 3. ed. Porto Alegre: Bookman, 2001. 719 p.

MCATAMNEY, Lynn; CORLETT, E. Nigel. RULA: a survey method for the investigation of world-related upper limb disorders. Applied Ergonomics, Nottingham, v. 24, n. 2, p. 91-99, 1993. Disponível em: <http://www.eng.auburn.edu/ise/courses/insy3020/RULA.pdf> Acesso em: 12 abril 2014.

OLIVEIRA, José Teotônio de. LER - lesão por esforços repetitivos um conceito falho e prejudicial. Arq Neuropsiquiatr, Belo Horizonte, v. 57, n. 1, p. 126-131, 1999. Disponível em: <http://www.scielo.br/pdf/anp/v57n1/1551.pdf> Acesso em: 01 maio 2014.

OHNO, Taiichi. O sistema Toyota de produção: além da produção em larga escala. Porto Alegre: Bookman, 1997. 149 p.

ORMELEZ, Camilla R.; ULBRICHT, Leandra. Análise ergonômica do trabalho aplicada a um posto de trabalho com sobrecarga física. Revista Uniandrade, Curitiba, v.11, n.2, Jul./dez. 2010. 
PAIVA, Ely Laureano; CARVALHO Jr, José Mário; FENSTERSEIFER, Jaime Evaldo Estratégias de produção e de operações: conceitos, melhores práticas, visão de futuro. Porto Alegre: Bookman, 2004. 192 p.

PIZO, Carlos A.; MENEGON, Nilton L. Análise ergonômica do trabalho e o reconhecimento científico do conhecimento gerado. Produção, Maringá, v. 20, n. 4, p. 657-668, out./dez. 2010. Disponível em: <http://www.scielo.br/pdf/prod/v20n4/AOP_200902028.pdf>Acesso: 30 mar. 2014.

POLONI, Gabriela P.; SILVA, Luciana C. L. Estudo de produtividade na estamparia de terminais MQS (micro quadlock system) em uma autopeça de grande porte. In: XXX ENCONTRO NACIONAL DE ENGENHARIA DE PRODUÇÃO, 2010, São Carlos. Anais eletrônicos ENEGEP 2010. p. 1-14. Disponível em: <http://www.abepro.org.br/biblioteca/enegep2010_TN_STP_113_745_15153.pdf>Acesso em: 28 março 2014.

PRODANOV, Cleber C; FREITAS, Ernani C. de. Metodologia do trabalho científico: métodos e técnicas da pesquisa e do trabalho acadêmico. 2.ed. Novo Hamburgo: Feevale, 2013. 277 p.

PUENTES-LAGOS, David E.; GARCÍA-ACOSTA, Gabriel. Tecnología y pensamiento futuro del trabajo desde la ergonomía en momentos de crisis global. Revista de Salud Pública, Bogotá, v. 14, sup (1), p. 122-137, Junio 2012. Disponível em: <http://www.scielo.org.co/pdf/rsap/v14s1/v14s1a11.pdf> Acesso em: 31 março 2014.

RABY, Françoise; BAILLE, Jacques; BRESSOUX, Pascal; CHAPELLE, Carol . Ergonomic theory and practice: What language learners do in a self-access room. La Reveu de Geras, Paris, n. 41-42, p. 67-84, 2003. Disponível em: <http://asp.revues.org/1175> Acesso em: 31 março 2014.

RENNER, Jacinta S. Prevenção de distúrbios osteomusculares relacionados ao trabalho. Boletim da Saúde, Porto Alegre, v.19, n.1, p. 73-80, jan./jun. 2005.

RIBEIRO, Herval Pina. A violência oculta do trabalho: as lesões por esforços repetitivos. Rio de Janeiro: Editora Fiocruz, 1999. 240 p.

ROBERTSON, Michelle M. Macroergonomics: a work system design perspective. In: 2001 CONFERENCE - ERGONOMICS FOR CHANGING WORK, 2001, Montreal. Proceedings of the SELF-ACE. Montreal. p. 67-77. Disponível em: <http://www.ergonomieself.org/documents/36eme-Montreal-2001/PDF-ENG/v1-07b-ROBERTSON.pdf> Acesso em: 26 abril 2014.

ROETTING, Matthias; LUCZAK, Holger. Ergonomics as Integrating Constituent in Occupational Safety and Health-Past, Present, and Future, International Journal of Occupational Safety and Ergonomics, Hopkinton, v. 7, n. 4, p. 507-526, 2001. Disponível em: <http://www.ciop.pl/805> Acesso em: 31 março 2014.

SAGOT, Jean-Claude; GOUIN, Valerie; GOMES, Samuel. Ergonomics in product design: safety factor. Safety Science, Belfort, v. 41, p. 137-154, 2003.. Disponível em: <www.elsevier.com/locate/ssci> Acesso em: 31 março 2014. 
SEMENSATO, Bárbara Ilze. Análise comparativa entre as metodologias de pesquisa científica e as metodologias da ação ergonômica a partir de um constructo teórico. Ação Ergonômica, v. 8, n.1, p. 33-47, 2013..

SHERIDAN, Thomas B. Evaluating models in systems ergonomics with a taxonomy of model attributes. Applied Ergonomics, Cambridge, v. 45, p. 78 - 84, 2014.. Disponível em: $<$ http://ac.els-cdn.com/S0003687013000598/1-s2.0-S0003687013000598main.pdf? tid=dc1ac306-c1d9-11e3-a08f00000aab0f26\&acdnat=1397262899_17f4a3f024dfbf9658fdf39c5> Acesso em: 21 abril 2014. DOI:http://dx.doi.org/10.1016/j.apergo.2013.03.018.

SHINGO, Shigeo, O sistema Toyota de produção: do ponto de vista da engenharia de produção. Porto Alegre: Bookman, 1996. 291 p.

SLEATOR, Alex; GORE, Donna; VIDLER, Graham. Work Related Upper Limb Disorders. London: RESEARCH PAPER, 1998. 38 p. Disponível em:

<http://www.parliament.uk/documents/commons/lib/research/rp98/rp98-051.pdf> Acesso em: 12 abril 2014.

SYVERSON, Chad. What Determines Productivity? Journal of Economic Literature, Chicago, v. 49, n. 2, p. 326-365, 2011. Disponível em:

<http://faculty.chicagobooth.edu/chad.syverson/research/productivitysurvey.pdf> Acesso em: 29 março 2014.

TANGEN, Stefan. Understanding the concept of productivity. In: 7th Asia Pacific Industrial Engineering and Management Systems Conference, 2002, Taipei. Proceedings of the 7th Asia Pacific Industrial Engineering and Management Systems. Stockholm: Dept. of Production Engineering, The Royal Institute of Technology, 2002. Disponível em: < http://www.aipa.ca/wp-content/uploads/2013/11/pap_Tangen2002-

UnderstandingTheConceptOfProductivity.pdf> Acesso em: 27 julho 2014.

THEBERGE, N.; NEUMANN, W. P. Doing 'organizational work': Expanding the conception of professional practice in ergonomics. Applied Ergonomics, Ontario, v. 42, p. 76-84, 2010. Disponível em: <http://ac.els-cdn.com/S0003687010000700/1-s2.0-S0003687010000700main.pdf?_tid=cee69cf4-c1da-11e3-a4ea-

00000aab0f26\&acdnat=1397263307_e95fbd9be4c6fc68951723b6fd246843>. Acesso em: 11 abril 2014.

THIOLLENT, Michel. Pesquisa-ação nas organizações. São Paulo: Editora Atlas, 1997. $164 \mathrm{p}$.

TOFT, Yvonne; HOWARD, Prue; JORGENSEN, David. Human-centred engineers-a model for holistic interdisciplinary communication and professional practice. International Journal of Industrial Ergonomics, Queensland, v. 31, p. 195-2002, 2003. Disponível em:

<http://www.sciencedirect.comscience/journal/01698141/31/3> Acesso em: 31 março 2014.

TORMAN, Vanessa B. L.; COSTER, Rodrigo; RIBOLDI, João. Normalidade de variáveis: métodos de verificação e comparação de alguns testes não-paramétricos por simulação. Revista HCPA, Porto Alegre, v. 32, n. 2, p. 227-234, 2012. Disponível em: <http://seer.ufrgs.br/hcpa> Acesso em: 5 dez. 2014.

VINK, Peter; KONINGSVELD, Ernst A.P.; MOLENBROEK, Johan F. Positive outcomes of participatory ergonomics in terms of greater comfort and higher productivity. Applied 
Ergonomics, Amsterdam, v. 37, p. 537-546, 2006. Disponível em: <http://ac.elscdn.com/S0003687006000597/1-s2.0-S0003687006000597-main.pdf?_tid=a13197b8-bc5911e3-8feb-00000aab0f01\&acdnat=1396658069_899025a89581ad4dfde42e5ed4986ba4> Acesso em: 4 abril 2014.

WILSON, John R. Fundamentals of ergonomics in theory and practice. Applied Ergonomics, Nottingham v. 31, p. 557 - 567, 2000. Disponível em:

<http://25aban.com/my_doc/imensepahan/my_articles/Fundamentals\%20of\%20ergonomics \%20in\%20theory\%20and\%20practice.pdf> Acesso em: 11 abril 2014.

WILSON, John R. Fundamentals of systems ergonomics/human factors. Applied Ergonomics, Nottingham, v. 45, p. 5-13, 2014. Disponível em: <http://ac.elscdn.com/S0003687013000628/1-s2.0-S0003687013000628-main.pdf?_tid=002bc0fa-c1d611e3-8160-00000aacb362\&acdnat $=1397261242$ de712ce5155bf5d7b922a1180fce0ac8 > Acesso em: 11 abril 2014.

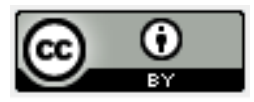

Artigo recebido em 30/09/2015 e aceito para publicação em 01/11/2016 DOI:http://dx.doi.org/ 10.14488/1676-1901.v16i4.2186 